\title{
Modulation of simple sinusoidal patterns by a coupled oscillator model for biped walking
}

\author{
Jun Morimoto ${ }^{1,2}{\text {, Gen } \text { Endo }^{2,3} \text {, Jun Nakanishi }}^{1,2}$, \\ Sang-Ho Hyon ${ }^{1,2}$, and Gordon Cheng ${ }^{1,2}$ \\ ${ }^{1}$ Computational Brain Project, ICORP, JST \\ ${ }^{2}$ ATR Computational Neuroscience Labs \\ ${ }^{3}$ Sony Intelligence Dynamics Laboratories, Inc. \\ 2-2-2 Hikaridai Seika-cho Soraku-gun Kyoto, 619-0288, JAPAN \\ Email: xmorimo@atr.jp
}

\begin{abstract}
We show that a humanoid robot can step and walk using simple sinusoidal desired joint trajectories with their phase adjusted by a coupled oscillator model. We use the center of pressure location and velocity to detect the phase of the lateral robot dynamics. This phase information is used to modulate the desired joint trajectories. We applied the proposed control approach to our newly developed human sized humanoid robot and a small size humanoid robot developed by Sony, enabling them to generate successful stepping and walking patterns.
\end{abstract}

\section{INTRODUCTION}

Many biped walking studies have emphasized that humanoid robots have inverted pendulum dynamics (Fig. 3), with the top at the center of mass and the base at the center of pressure, and proposed control strategies to stabilize the dynamics [1]-[3].

In this study we propose using the center of pressure to detect the phase of the inverted pendulum dynamics (Fig. 3 ). We use simple periodic functions (sinusoids) as desired joint trajectories. We show that synchronization of the desired trajectories at each joint with the inverted pendulum dynamics can generate stepping and walking.

Related work: Because biped walking is a periodic movement, it has been suggested that methods to synchronize biped controllers with the robot dynamics are useful to generate stable walking patterns. Several studies designed walking trajectories as a function of a physical variable of the robot (e.g. ankle joint angle) [4]-[6]. There is also growing interest in biologically inspired locomotion control using a neural oscillator [7]-[9] or using a phase oscillator model with phase reset methods [10], [11]. These studies mainly used foot contact information or ground reaction force in exploiting the entrainment property of the neural or phase oscillator models.

To the best of our knowledge, this study is the first attempt to apply an oscillator model to a human sized humanoid robot (Fig.1(left)) for biped walking in a real environment. We also apply our method to the Sony humanoid robot (Fig.2).

\section{CONTROL APPROACH}

Our biped control approach uses a coupled phase oscillator model to modulate simple sinusoidal patterns. The aim of

\author{
Darrin Bentivegna and Christopher G. Atkeson \\ The Robotics Institute, Carnegie Mellon University \\ 5000 Forbes Ave., Pittsburgh, PA, 15213, USA
}

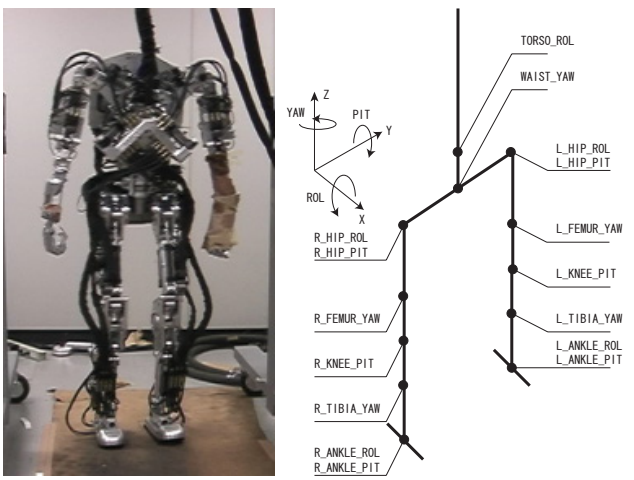

Fig. 1. (Left) Our human sized hydraulic humanoid robot developed by SARCOS. height: $1.59 \mathrm{~m}$, total weight: $72 \mathrm{~kg}$. (Right) Simple 3D biped simulation model.

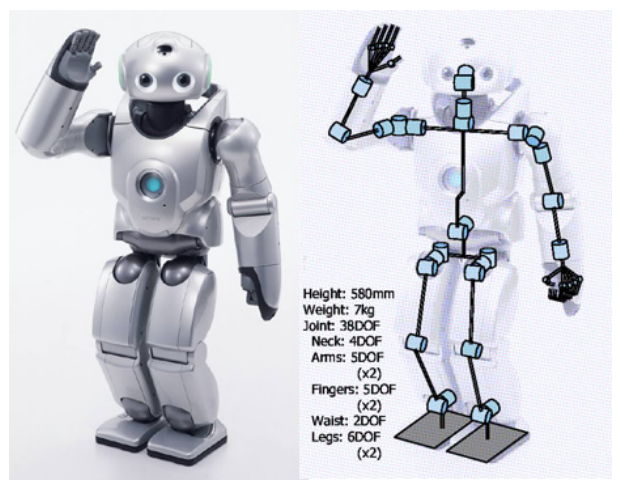

Fig. 2. Sony humanoid robot

using the coupled phase oscillator model is to synchronize periodic patterns generated by the controller with the dynamics of the robot. First, we briefly explain the phase oscillator model in section II-A and phase coordination for the biped walking task in section II-C. To use the coupled phase oscillator model, we need to detect the phase of the robot. We introduce our method to detect the robot phase in section II-B. In this study, we propose using simple sinusoidal patterns as nominal trajectories at each joint. We describe the design of the nominal trajectories for stepping movement in section IID. We also introduce a stabilizing controller which takes the 
maximum body roll angle as input state and modulates the amplitude of the nominal sinusoidal patterns in section II-E. In section II-F, we describe how we use additional nominal trajectories to make forward movements for biped walking.

\section{A. Coupled oscillator model}

Here, we consider the behavior of the following dynamics of the phase of a biped controller $\phi_{c}$ and the phase of the robot dynamics $\phi_{r}$ :

$$
\begin{gathered}
\dot{\phi}_{c}=\omega_{c}+K_{c} \sin \left(\phi_{r}-\phi_{c}\right), \\
\dot{\phi}_{r}=\omega_{r}+K_{r} \sin \left(\phi_{c}-\phi_{r}\right),
\end{gathered}
$$

where $\omega_{c}$ and $\omega_{r}>0$ are natural frequencies of the controller and the robot dynamics, and $K_{c}, K_{r}$ are positive coupling constants. Then, the oscillators run with the phase difference: $\Psi^{*}=\phi_{r}-\phi_{c}=\frac{\omega_{r}-\omega_{c}}{K_{c}+K_{r}}$ and the coupled frequency: $\omega^{*}=$ $\frac{K_{r} \omega_{c}+K_{c} \omega_{r}}{K_{c}+K_{r}}$ when they are entrained. However, usually we do not have exact information of the natural frequency of the robot dynamics and the coupling constant $K_{r}$ in Equation (2). In this study, we use Equation (1) to synchronize the phase of controller $\phi_{c}$ with the phase of the robot dynamics $\phi_{r}$.

\section{B. Phase detection from the robot dynamics}

As previous studies have pointed out, controlling the inverted pendulum dynamics represented by the center of mass and the center of pressure (Fig. 3) is a major issue in controlling biped robots. The inverted pendulum dynamics has a four dimensional state space $\mathbf{x}=(x, \dot{x}, \psi, \dot{\psi})$. In this study, we decided to use the center of pressure $x$ and the velocity of the center of pressure $\dot{x}$ to detect the phase of the robot dynamics:

$$
\phi_{r}(\mathbf{x})=-\arctan \left(\frac{\dot{x}}{x}\right) .
$$

\section{Phase coordination}

In this study, we use four oscillators with phases $\phi_{c}^{i}$, where $i=1,2,3,4$. We introduce coupling between the oscillators and the phase of the robot dynamics $\phi_{r}(\mathbf{x})$ to regulate the desired phase relationship between the oscillators as in (1):

$$
\dot{\phi}_{c}^{i}=\omega_{c}+K_{c} \sin \left(\phi_{r}(\mathbf{x})-\phi_{c}^{i}+\alpha_{i}\right),
$$

where $\alpha_{i}$ is the desired phase difference. We use four different phase differences, $\boldsymbol{\alpha}=\left\{\alpha_{1}, \alpha_{2}, \alpha_{3}, \alpha_{4}\right\}=\left\{0.0, \frac{1}{2} \pi, \pi, \frac{3}{2} \pi\right\}$, to make symmetric patterns for a stepping movement by left and right limbs (see section II-D.2), and also to make symmetric patterns for a forward movement by left and right limbs (see section II-F).

\section{Stepping controller for lateral movement}

1) Side-to-side controller for lateral movement: First, we introduce a controller to generate side-to-side movement. We use the hip joints $\theta_{\text {hip_r }}$ and the ankle joints $\theta_{\text {ankle_r }}$ (Fig. 4(left)) for the movement. Desired joint angles for each joint are:

$$
\begin{array}{r}
\theta_{\text {hip_r } r}^{d}\left(\phi_{c}\right)=A_{h i p_{-} r} \sin \left(\phi_{c}\right), \\
\theta_{\text {ankle_r } r}^{d}\left(\phi_{c}\right)=-A_{\text {ankle_r } r} \sin \left(\phi_{c}\right),
\end{array}
$$
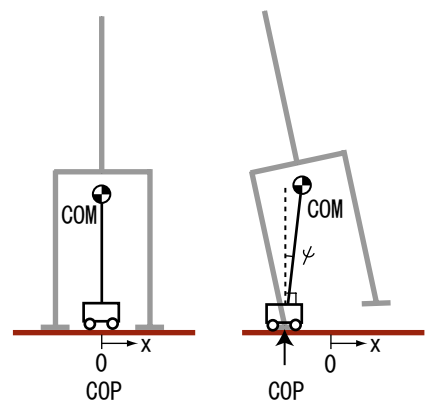

Fig. 3. Inverted pendulum model represented by the center of pressure (COP) and the center of mass $(\mathrm{COM})$

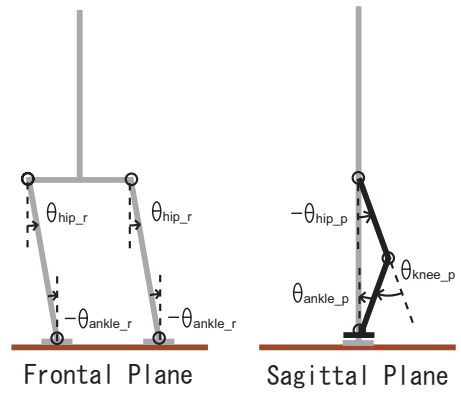

Fig. 4. Stepping controller: (left) Controller for side-to-side movement. (right) Controller for foot clearance.

where $A_{h i p_{-} r}$ and $A_{a n k l e \_}$are the amplitudes of a sinusoidal function for side-to-side movements at the hip and the ankle joints, and we use a oscillator with the phase $\phi_{c}=\phi_{c}^{1}$.

2) Vertical foot movement to make clearance: To achieve foot clearance, we generate vertical movement of the feet (Fig. 4(right)) by using simple sinusoidal trajectories:

$$
\begin{aligned}
\theta_{\text {hip_p }}^{d}\left(\phi_{c}\right) & =A_{p} \sin \left(\phi_{c}\right)+\theta_{\text {hip_p }}^{\text {res }}, \\
\theta_{\text {knee_p }}^{d}\left(\phi_{c}\right) & =-2 A_{p} \sin \left(\phi_{c}\right)+\theta_{\text {knee_p }}^{\text {res }}, \\
\theta_{\text {ankle_p }}^{d}\left(\phi_{c}\right) & =-A_{p} \sin \left(\phi_{c}\right)+\theta_{\text {ankle_p }}^{\text {res }},
\end{aligned}
$$

where $A_{p}$ is the amplitude of a sinusoidal function to achieve foot clearance, $\theta_{\text {hip }}^{\text {res }}$,,$\theta_{\text {knee_ }}^{\text {res }}$, $\theta_{\text {ankle_p }}^{\text {res }}$ represent the rest posture of the hip, knee, and ankle joints respectively (Fig. 4 (right)). We use the oscillator with phase $\phi_{c}=\phi_{c}^{1}$ for right limb movement and use the oscillator with phase $\phi_{c}=\phi_{c}^{3}$, which has phase difference $\phi_{c}^{3}=\phi_{c}^{1}+\pi$, for left limb movement.

\section{E. Stabilizing Controller}

So far, we have discussed using coupled oscillators to synchronize the controller with the robot dynamics. Here we introduce a controller to stabilize the robot.

We use body roll angle $\theta_{\text {roll }}$ (Fig. 5) which can be detected by sensors on the robot. We focus on the body roll $\theta_{\text {roll }}=$ $\theta_{\text {roll_max }}$ at its extremes $\left(\dot{\theta}_{\text {roll }}=0\right)$. We have designed a stabilizing controller:

$$
A_{f b}=-K_{f b}\left(\theta_{\text {roll_max }}-\theta_{\text {roll_max }}^{d}\right),
$$

where the gain $K_{f b}$ can be derived by solving a linear quadratic regulator optimization problem, and $A_{f b}$ is the 


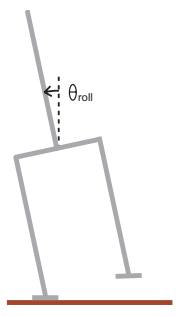

Fig. 5. Using roll angle to stabilize stepping and walking patterns feedback amplitude. The feedback amplitude $A_{f b}$ modulates the amplitude of the ankle joints for side-to-side movements. Equation (6) becomes:

$$
\theta_{\text {ankle_r }}^{d}\left(\phi_{c}\right)=-\left(A_{\text {ankle_r }}+A_{f b}\right) \sin \left(\phi_{c}\right) .
$$

We approximate a return map for the maximum roll angle $\theta_{\text {roll_max }}$ by a linear dynamics model:

$$
\theta_{\text {roll_max }}(k+1)=H \theta_{\text {roll_max }}(k)+B A_{f b}(k),
$$

We find the linear feedback controller which maximizes an objective function:

$$
J=\sum_{k}^{\infty} Q\left(\theta_{\text {roll_max }}(k)-\theta_{\text {roll_max }}^{d}\right)^{2}+R A_{f b}^{2},
$$

where $Q=1.0$ and $R=0.1$ are parameters of the objective function. We collected data to approximate the parameters $H$ and $B$ of the linear dynamics by using random feedback amplitudes $A_{f b}$.

\section{F. Additional controller for biped walking}

To walk forward, we use an additional sinusoidal trajectory:

$$
\begin{aligned}
& \theta_{\text {hip_s }}^{d}\left(\phi_{c}\right)=A_{\text {hip_s } s} \sin \left(\phi_{c}\right), \\
& \theta_{\text {ankle_s }}^{d}\left(\phi_{c}\right)=-A_{\text {ankle_s }} \sin \left(\phi_{c}\right) \text {, }
\end{aligned}
$$

where $A_{\text {hip_s }}$ and $A_{\text {ankle_s }}$ are amplitudes of sinusoidal functions at the hip and the ankle joints for biped walking, and we use the phase $\phi_{c}=\phi_{c}^{2}$ which has $\frac{1}{2} \pi$ phase difference with $\phi_{c}^{1}$ for right limb and use the phase $\phi_{c}=\phi_{c}^{4}$ which has $\pi$ phase difference with $\phi_{c}^{2}$. The desired nominal trajectories for right hip and ankle pitch joints become:

$$
\begin{aligned}
& \theta_{\text {hip_p }}^{d}=A_{p} \sin \left(\phi_{c}^{1}\right)+A_{\text {hip_s } s} \sin \left(\phi_{c}^{2}\right)+\theta_{\text {hip_p }}^{\text {res }}, \\
& \theta_{\text {ankle_p }}^{d}=-A_{p} \sin \left(\phi_{c}^{1}\right)-A_{\text {ankle_s }} \sin \left(\phi_{c}^{2}\right)+\theta_{\text {ankle_p }}^{\text {res }}
\end{aligned}
$$

where we use $\phi_{c}^{3}$ and $\phi_{c}^{4}$ for left limb instead of $\phi_{c}^{1}$ and $\phi_{c}^{2}$.

\section{SIMULATION}

We applied our proposed method to a simple 3D biped robot model (Fig. 1(right)) (height: $1.59 \mathrm{~m}$, total weight: $20 \mathrm{~kg}$ ).

\section{A. Stepping movement using the coupled oscillator model}

We applied our proposed method to generate stepping movements. We used the amplitudes $A_{h i p_{-} r}=3.0 \mathrm{deg}$ and $A_{\text {ankle_r }}=3.0 \mathrm{deg}$ for the side-to-side movement, and $A_{p}=3.5 \mathrm{deg}$ to make foot clearance. We used the coupling constant $K_{c}=9.4$. We set the natural frequency of the controller to $\omega_{c}=3.6 \mathrm{rad} / \mathrm{sec}$. By only using simple sinusoidal

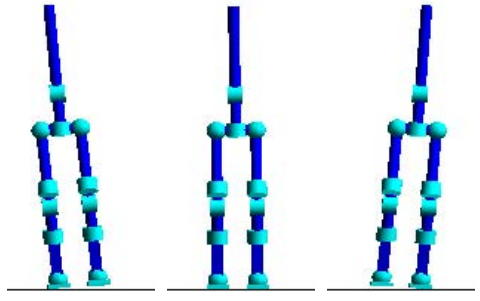

Fig. 6. Stepping movement using coupled oscillator: (left) $\phi_{c}^{1}=\frac{1}{2} \pi \mathrm{rad}$ (center) $\phi_{c}^{1}=0 \mathrm{rad}$ (right) $\phi_{c}^{1}=\frac{3}{2} \pi \mathrm{rad}$.

trajectories at each joint without using a coupled oscillator model could not make stepping movement (i.e., could not produce a single support phase. See a profile of the center of pressure in figure 9(left)). On the other hand, by using the proposed phase oscillator model with the simple sinusoidal trajectories to phase lock the trajectories to the oscillation of the robot, we successfully generated stepping movement (Fig. 6). Figure 7 shows trajectories of desired and actual hip joint angles $\theta_{\text {hip_r }}^{d}, \theta_{\text {hip_r }}$ (Fig. 4(left)). Figure 7(left) represents the result of without using a coupled oscillator model. The desired trajectory is the same as the original simple sinusoidal trajectory. Figure 7(right) represents the result of using a coupled oscillator model. The desired trajectory is modulated by the coupling with the phase of the robot dynamics $\phi_{r}(\mathbf{x})$. This modulated trajectory made stepping movement possible. Averaged frequency with coupling was $\dot{\phi}_{c}^{a v}=\frac{1}{T}\left(\phi_{c}(T+t)-\right.$ $\left.\phi_{c}(t)\right)=4.0 \mathrm{rad} / \mathrm{sec}$, where $T$ is a stepping period. Note that $\dot{\phi}_{c}$ is not constant because of the coupling.

To show how the coupled oscillator model in equation (1) and (2) worked with the biped robot model, we tested a different controller with a different natural frequency, $\omega=2.5$ $\mathrm{rad} / \mathrm{sec}$. Although the natural frequency was different (see Figure 7(left) and Figure 8(left)), the modulated averaged frequency $\dot{\phi}_{c}^{a v}=3.9 \mathrm{rad} / \mathrm{sec}$ was similar to the previous averaged frequency, $\dot{\phi}_{c}^{a v}=4.0 \mathrm{rad} / \mathrm{sec}$ (see Figure 7(right) and Figure 8(right)). By considering the coupled frequency $\omega^{*}$ introduced in section II-A, this result indicates that the current coupling constant $K_{c}=9.4$ is large enough to make the controller frequency close to the natural frequency of the robot dynamics.

Figure 9 shows the time course of the center of pressure with (right) and without (left) coupling. Figure 9(right) shows that the simulated robot successfully generated dynamic stepping.

Figure 10 shows sequences of maximum body roll $\theta_{\text {roll_max }}$. We used three different desired maximum body roll angles $\theta_{\text {roll }}^{d}=r_{d}=3.5,4.0$, and 4.5 rad. Results shows that the actual maximum body roll $\theta_{\text {roll_max }}$ can converge close to the desired target.

We need rapid movement of the center of pressure during the double support phase for dynamic biped walking [12]. Although we did not explicitly specify the trajectory of the center of pressure to move fast during the double support phase, the coupling with the phase detected from the center of pressure implicitly generates the trajectory suitable for dynamic biped walking (Fig. 9(Right)). 

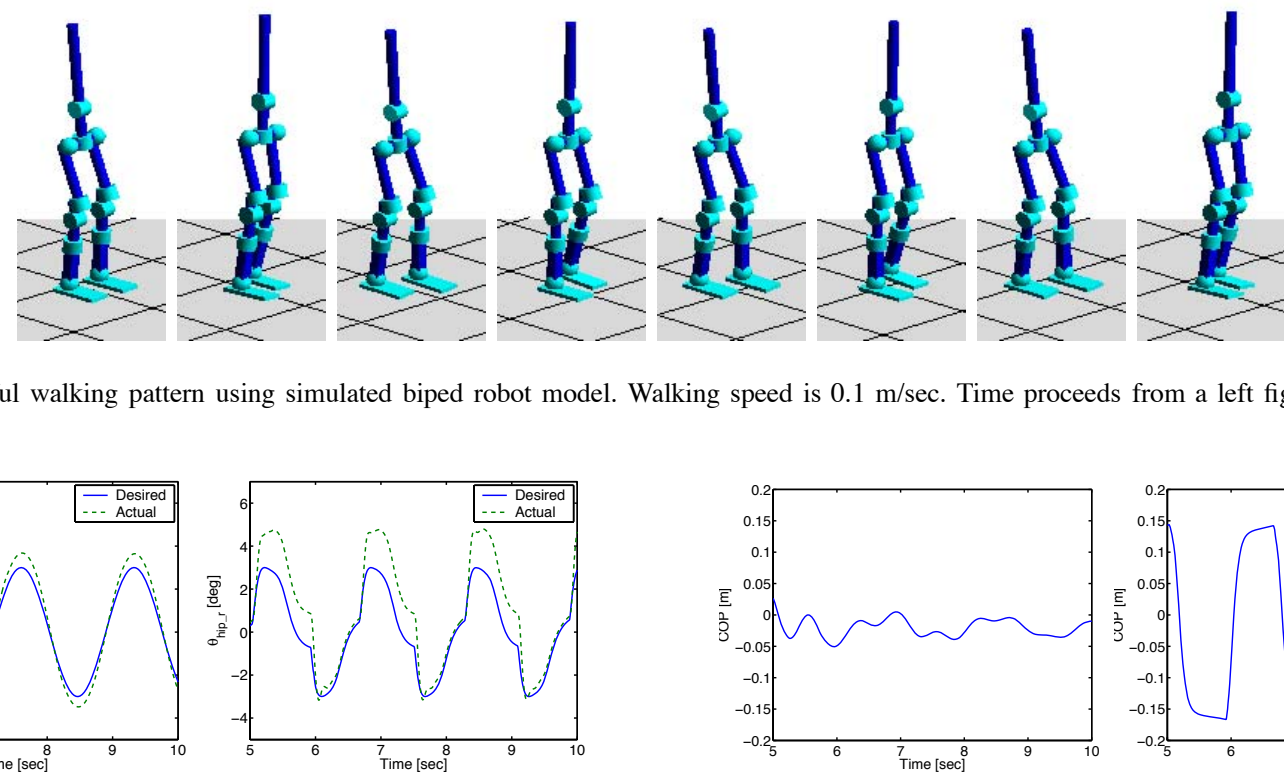

Fig. 11. Successful walking pattern using simulated biped robot model. Walking speed is $0.1 \mathrm{~m} / \mathrm{sec}$. Time proceeds from a left figure to a right figure
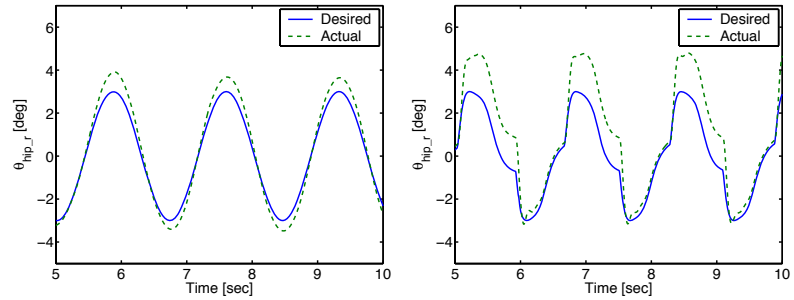

Fig. 7. Generated desired and actual trajectory at hip joint (Left) Not using coupled oscillator. (Right) Using coupled oscillator.
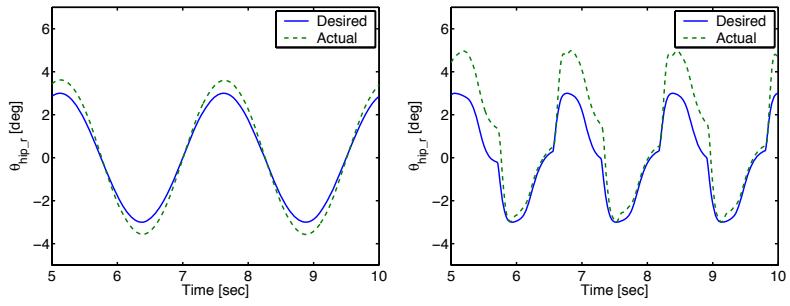

Fig. 8. Generated desired and actual trajectory at the hip joint for a slower controller frequency of $\omega=2.5 \mathrm{rad} / \mathrm{sec}$. (Left) Not using coupled oscillator. (Right) Using coupled oscillator.

\section{B. Biped walking}

Here, we used the additional sinusoidal pattern introduced in section II-F. We set the amplitude parameter as $A_{\text {hip_s }}=3.0$ $\mathrm{rad}$ and $A_{\text {ankle_s }}=2.0 \mathrm{rad}$. Figure 11 shows the successful walking pattern generated by our control approach.

We calculated the ratio between the single support phase and double support phase. The ratio was around $\frac{T_{\text {double }}}{T_{\text {double }}+T_{\text {single }}} \times$ $100=20 \%$, where $T_{\text {double }}$ is a period of time of double support phase and $T_{\text {single }}$ is a period of time of single support phase in a walking cycle. This ratio is similar to the ratio for human biped walking. This ratio is achieved by our proposed controller without using pre-designed trajectories of the center of pressure.

\section{EXPERIMENTAL RESULT}

\section{A. Application to human sized humanoid robot}

1) Stepping movement: We applied the proposed stepping method to a human sized humanoid robot (Fig. 1(left)). We used the amplitudes $A_{\text {hip_r }}=3.0 \mathrm{rad}, A_{\text {ankle_r }}=3.0 \mathrm{rad}$, and $A_{p}=3.5 \mathrm{rad}$ for the sinusoidal patterns to generate side-to-side movement and foot clearance. We set the natural frequency of the controller to $\omega_{c}=3.14 \mathrm{rad} / \mathrm{sec}$ and set the coupling constant to $K_{c}=9.4$.
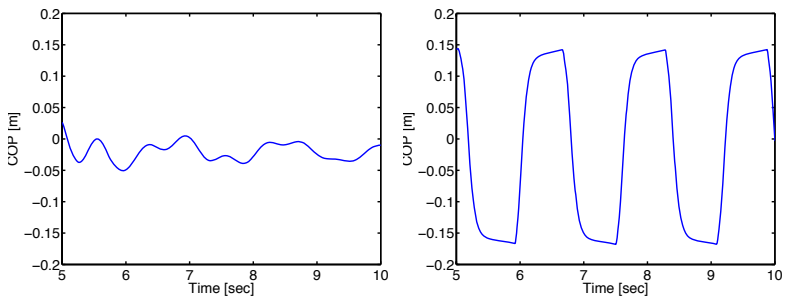

Fig. 9. Time course of the center of pressure of stepping movement. (Left) Not using coupled oscillator. (Right) Using coupled oscillator.

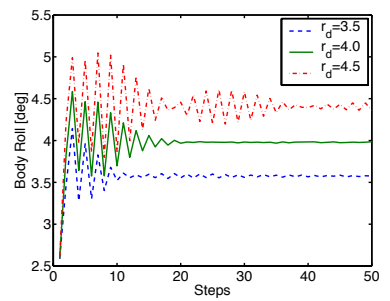

Fig. 10. Stabilized roll movement using the linear quadratic regulator

We could not approximate the $\theta_{\text {roll_max }}$ return map by a simple linear model in the real environment probably because of disturbances generated by the hydraulic cables. Instead, we manually designed parameters for the linear feedback controller in equation (10) with the parameter $K_{f b}=0.3$ and $\theta_{\text {roll_max }}^{d}=2.0 \mathrm{deg}$.

Here, we used an approximate center of pressure: $x=$ $\frac{x_{\text {foot }}^{l} F_{z}^{l}+x_{\text {foot }}^{r} F_{z}^{r}}{F_{z}^{r}+F_{z}^{l}}$, where $F_{z}^{l}$ and $F_{z}^{r}$ represent the left and right ground reaction force respectively, and $x_{\text {foot }}^{l}$ and $x_{\text {foot }}^{r}$ are the lateral position of each foot. We assume that feet are symmetrically placed $-x_{\text {foot }}^{l}=x_{\text {foot }}^{r}$. Because we only use this center of pressure to detect the phase of the robot dynamics $\phi_{r}(\mathbf{x})$ in equation (3), the scale of the foot position $x_{\text {foot }}^{l}$ and $x_{\text {foot }}^{r}$ can be arbitrary. We simply set $x_{\text {foot }}^{l}=$ $-x_{\text {foot }}^{r}=1.0 \mathrm{~m}$. Figure 13 shows the time course of the approximate center of pressure. Our proposed method successfully generates stepping movements (Fig. 12) with the profile of the center of pressure in figure 13.

2) Biped walking: We applied our proposed walking method to our newly developed humanoid robot (Fig. 1). We used the amplitudes $A_{\text {hip_s }}=4.0 \mathrm{rad}$ and $A_{\text {ankle_s }}=2.0 \mathrm{rad}$ for the additional movement in the sagittal plane.

Figure 14 shows the successful walking pattern of our humanoid robot. Our proposed method was able to generate 

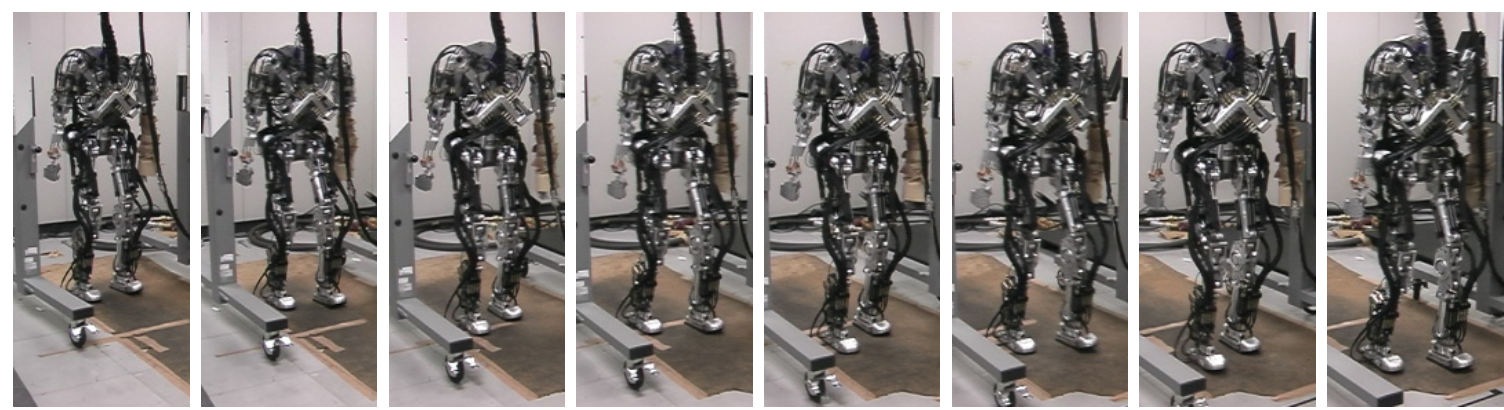

Fig. 14. Successful walking pattern of our humanoid robot. Time proceeds from a left figure to a right figure.
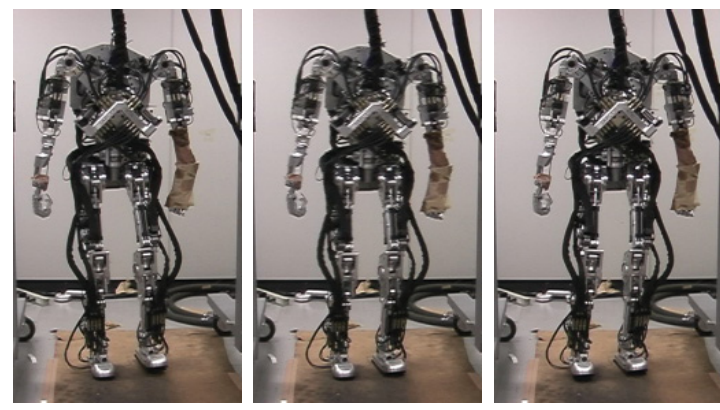

Fig. 12. Successful stepping of our humanoid robot: (left) $\phi_{c}^{1}=\frac{1}{2} \pi$, (center) $\phi_{c}^{1}=0.0$, (right) $\phi_{c}^{1}=\frac{3}{2} \pi$.

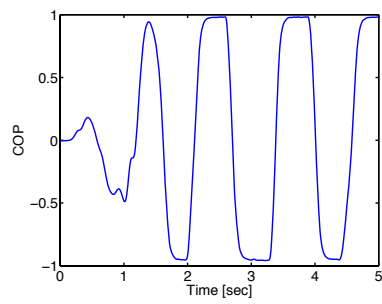

Fig. 13. Time course of the center of pressure of stepping movement in the real environment. Note that the trajectory does not perfectly reach 1 and -1 because there are sensor offsets of force sensors on soles.

successful walking patterns even in the real environment. Note that the black tube from the top of each photo in figure 14 is a hydraulic cable. The humanoid robot was not supported by the cable.

\section{B. Application to the Sony humanoid robot}

1) Stepping movement: We applied the proposed stepping method to the Sony humanoid robot. We set the parameters for the sinusoidal patterns to generate side-to-side movement and foot clearance as $A_{\text {hip_r }}=2.5 \mathrm{rad}, A_{\text {ankle_r }}=7.5 \mathrm{rad}$, and $A_{p}=3.5 \mathrm{rad}$. We used different phase difference $\alpha_{i}^{\prime}=\alpha_{i}-\frac{\pi}{3}$ instead of $\alpha_{i}$ in (4) because of actuator performance. We set the natural frequency of the controller to $\omega_{c}=6.28 \mathrm{rad} / \mathrm{sec}$ and set the coupling constant to $K_{c}=9.4$. For the Sony humanoid robot, we did not have to use the feedback controller in Equation (10) to make stepping and walking because the robot has wider foot relative to the height of the robot. We used the same approximate center of pressure to detect the phase of the robot dynamics. Figure 15 shows successful stepping of the Sony humanoid robot.
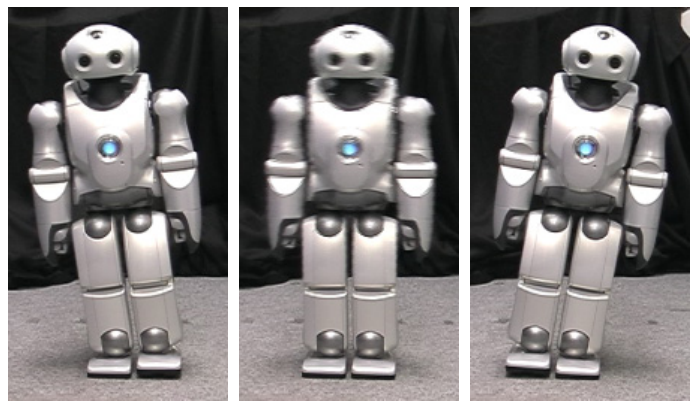

Fig. 15. Successful stepping of the Sony humanoid robot: (left) $\phi_{c}^{1}=\frac{1}{2} \pi$ $\operatorname{rad}($ center $) \phi_{c}^{1}=0 \mathrm{rad}$ (right) $\phi_{c}^{1}=\frac{3}{2} \pi \mathrm{rad}$.

2) Biped walking: We applied our proposed walking method to the Sony humanoid robot. We set the parameters for the additional movement as $A_{\text {hip_s }}=12.0 \mathrm{rad}$ and $A_{\text {ankle_s }}=8.0 \mathrm{rad}$. Figure 16 shows the successful walking pattern of the Sony humanoid robot. Our proposed method was able to generate successful walking patterns for robots of different sizes. Because our method to design a biped walking controller does not suffered from singularity problem at knee joints that comes from using inverse kinematics, knees can straighten during walking.

We also tested robustness of our biped controller using four different ground surfaces with different frictions. Each surface also has different height. The four surfaces include carpet with $0.0 \mathrm{~mm}$ (base level), plastic plate with $2.0 \mathrm{~mm}$, rubber plate with $3.5 \mathrm{~mm}$, and metal plate with $3.0 \mathrm{~mm}$. Figure 17 shows successful results of walking over the different surfaces without changing any parameter of the biped controller.

\section{DISCUSSION}

We proposed a method using the center of pressure position and velocity to detect the phase of the lateral robot dynamics. The detected phase of the robot lateral dynamics was used to modulate sinusoidal joint trajectories. The modulated trajectories enabled robots to generate successful stepping and walking patterns.

Because the angular frequency in Equation (4) is continuously changing during stepping and walking, not only the frequency of the controller changes toward the resonant frequency and excites the robot dynamics but also the time course of the sinusoidal patterns are modulated. Since the 

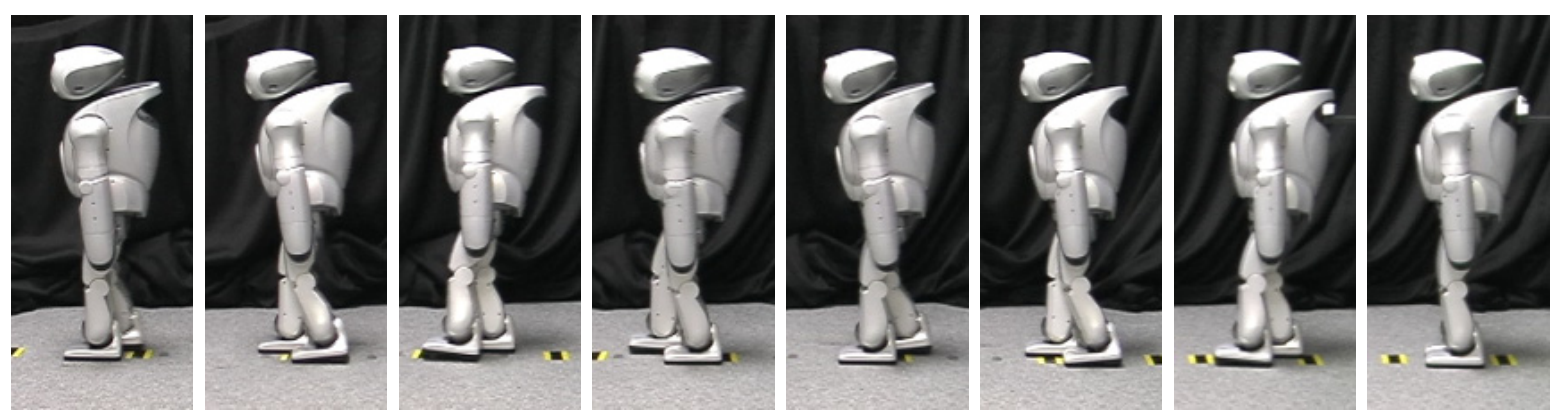

Fig. 16. Successful walking pattern of the Sony humanoid robot. Knees are stretched during walking. Time proceeds from a right figure to a left figure.
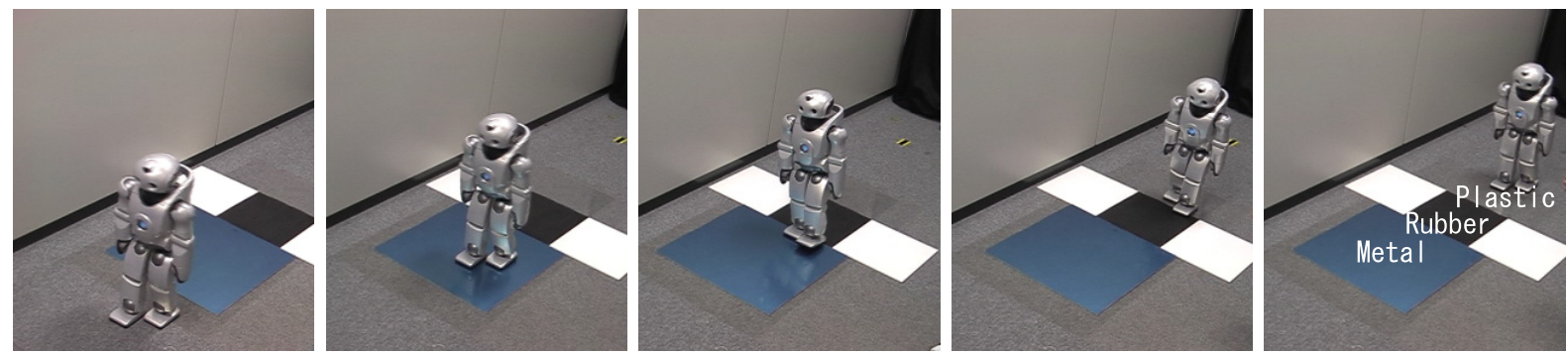

Fig. 17. Successful walking pattern of the Sony humanoid robot over four different surfaces: (1) Gray carpet, (2) Transparent plastic plate, (3) Black rubber plate, (4) Blue metal plate. Time proceeds from a right figure to a left figure.

center of pressure stops at outer edge of foot, the detected phase of the robot dynamics makes the phase of controller slow down during a single support phase. This phenomenon makes longer single support phases and made stepping and biped walking possible using our simulated and real humanoid robots.

As a comparison with previous approaches, we applied a phase reset method [10], [11] to stepping controller with our nominal trajectory. However, the simulated robot could not generate stepping movement because the phase reset does not modulate the nominal trajectory except at the timing of foot contact. Although the neural oscillator model has been successfully applied to the real robot applications [8], [9], we have to tune many parameters and nominal trajectories generated by the oscillator dynamics can be complicated.

Directly using the phase of the robot as the phase of the controller would be possible instead of using a coupled oscillator model in Equation (4). However, direct use of the robot dynamics does not enable us to modulate walking frequency by changing the natural frequency of the controller $\omega_{c}$ and coupling constant $K_{c}$ in Equation (4). Furthermore, directly using the detected phase which comes from sensor data can be susceptible to sensor noise.

We applied our proposed control approach to our newly developed human sized humanoid robot and the Sony humanoid robot.

In this study, we used a linear approximation of the return map of the maximum body roll angle to design stabilizing controllers for lateral movement. In the future, we will consider using a nonlinear return map model and optimization methods such as reinforcement learning or dynamic programming to acquire a nonlinear feedback controller, which can stabilize not only lateral movements, but sagittal movements as well in biped walking.

\section{REFERENCES}

[1] H. Miura and I. Shymoyama, "Dynamical walk of biped locomotion," Int. J. of Robotics Research, vol. 3, no. 2, pp. 60-74, 1984.

[2] K. Hirai, M. Hirose, and T. Takenaka, "The Development of Honda Humanoid Robot," in Proceedings of the 1998 IEEE International Conference on Robotics and Automation, 1998, pp. 160-165.

[3] K. Nagasaka, M. Inaba, and H. Inoue, "Stabilization of dynamic walk on a humanoid using torso position compliance control," in Proceedings of 17th Annual Conference on Robotics Society of Japan, 1999, pp. 11931194.

[4] S. Hyon and T. Emura, "Symmetric Walking Control: Invariance and Global Stability," in IEEE Int. Conf. on Robotics and Automation, Barcelona, Spain, pp. 1456-1462.

[5] M. Doi, Y. Hsegawa, and T. Fukuda, "Passive Trajectory Control of the Lateral Motion in Bipedal Walking," in IEEE Int. Conf. on Robotics and Automation, New Orleans, LA, USA, pp. 3049-3054.

[6] E. R. Westervelt, G. Buche, and J. W. Grizzle, "Inducing Dynamically Stable Walking in an Underactuated Prototype Planar Biped," in IEEE Int. Conf. on Robotics and Automation, New Orleans, LA, pp. 42344239.

[7] S. Miyakoshi, G. Taga, Y. Kuniyoshi, and A. Nagakubo, "Three dimensional bipedal stepping motion using neural oscillators -towards humanoid motion in the real world," in IEEE/RSJ Int. Conf. on Intelligent Robots and Systems, Victoria, Canada, 1998.

[8] G. Endo, J. Nakanishi, J. Morimoto, and G. Cheng, "Experimental Studies of a Neural Oscillator for Biped Locomotion with QRIO," in IEEE Int. Conf. on Robotics and Automation, Barcelona, Spain, pp. 598603.

[9] Y. Fukuoka and H. Kimura, "Adaptive Dynamic Walking of a Quadruped Robot on Irregular Terrain based on Biological Concepts," Int. Journal of Robotics Research, vol. 22, no. 2, pp. 187-202, 2003.

[10] J. Nakanishi, J. Morimoto, G. Endo, G. Cheng, S. Schaal, and M. Kawato, "Learning from demonstration and adaptation of biped locomotion," Robotics and Autonomous Systems, vol. 47, pp. 79-91, 2004.

[11] K. Tsuchiya, S. Aoi, and K. Tsujita, "Locomotion control of a biped locomotion robot using nonlinear oscillators," in Proceedings of the IEEE/RSJ International Conference on Intelligent Robots and Systems, Las Vegas, NV, USA, 2003, pp. 1745-1750.

[12] T. Sugihara and Y. Nakamura, "A Fast Online Gait Planning with Boundary Condition Relaxation for Humanoid Robots," in IEEE Int. Conf. on Robotics and Automation, Barcelona, Spain, pp. 306-311. 